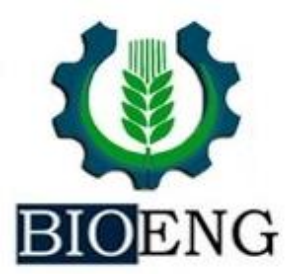

\title{
USO DE FERRAMENTA MOBILE NA AVALIAÇÃO DO BEM-ESTAR DE BOVINOS DE LEITE
}

A. J. Oliveira Júnior ${ }^{1 *}$, S. R. L. Souza ${ }^{1}$, V. C. Souza ${ }^{1}$, N. Z. Costa ${ }^{2}$, L. G. F. Bueno ${ }^{3}$, R. A. Almeida ${ }^{1}$

${ }^{1}$ UNESP - Faculdade de Ciências Agronômicas, Botucatu, SP, Brasil

${ }^{2}$ UNESP - Faculdade de Medicina Veterinária e Zootecnia, Botucatu, SP, Brasil

${ }^{3}$ UNESP - Faculdade de Medicina Veterinária e Zootecnia, Dracena, SP, Brasil

Article history: Received 25 May 2018; Received in revised form 28 August 2018; Accepted 18 September 2018; Available online 30 September 2018.

\section{RESUMO}

Os elementos climáticos podem influenciar o desempenho animal de forma negativa, desta maneira deve-se atuar no sentido de minimizar seus efeitos, utilizando construções adequadas para abrigá-los. As diferenças climáticas entre o país de origem dos bovinos leiteiros e o Brasil impedem que as vacas expressem todo o seu potencial genético para a produção de leite em função do estresse térmico, especialmente durante o verão, onde observam-se altas temperaturas e umidades relativas. O objetivo desse trabalho foi utilizar a ferramenta mobile Orvalho ${ }^{\circledR}$ para cálculo de índices de conforto visando o bem-estar de vacas leiteiras. Foram coletadas variáveis ambientais no galpão de descanso das vacas após ordenha, tais como: Temperatura de bulbo seco (Tbs), Temperatura de globo negro (Tgn) e Umidade relativa do ar (UR). Após a coleta dos dados ambientais, foi calculado o índice de conforto térmico ITGU e ITU através do aplicativo Orvalho ${ }^{\circledR}$ para inferir o estado de estresse térmico dos animais. Como resultado observou-se que os valores apresentados estão dentro dos limites de conforto para bovinos de leite, com ITGU máximo de 72,03 e ITU máximo de 72,46. O app Orvalho ${ }^{\circledR}$ auxiliou de forma prática o cálculo dos índices estudados, demonstrando ser um recurso móvel útil para pequenas avaliações de condições térmicas.

Palavras-chave: Ambiência, Zootecnia de precisão, Bovinos de leite, Conforto térmico, Android

\section{MOBILE TOOL IN EVALUATING THE WELFARE OF DAIRY CATTLE}

\begin{abstract}
The climatic elements can influence the animal performance in a negative way, in this way one must act in order to minimize its effects, using suitable constructions to shelter them. The climatic differences between the country of origin of dairy cattle and Brazil prevent cows from expressing their genetic potential for milk production as a function of thermal stress, especially during the summer, where high temperatures and relative humidity are observed air. The objective of this work was to use the mobile tool Dewel ${ }^{\circledR}$ to calculate comfort indexes aiming the welfare of dairy cows. Environmental variables were collected in the cow resting shed after milking, such as: Dry Bulb Temperature (Tbs), Black Globe Temperature (Tgn) and Relative Humidity (RH). After collecting the environmental data, the ITGU and ITU thermal comfort index was calculated through the Orvalho ${ }^{\circledR}$ application to infer the thermal

\footnotetext{
*arilsonjr@outlook.com

Trabalho apresentado no $3^{\circ}$ Seminário de Construção Rural e Ambiência Aplicados à Produção Animal, 2018.
} 
stress state of the animals. As a result, the values presented are within the limits of comfort for dairy cows (ITGU $\max =72,03$ and ITU $\max =72,46$ ). The Orvalho ${ }^{\circledR}$ app helped in a practical way the calculation of the indexes studied, proving to be a useful mobile resource for small evaluations of thermal conditions.

Keywords: Ambience, Animal science precision, Dairy cows, Thermal comfort, Android

\section{INTRODUÇÃO}

As instalações têm grande importância nas propriedades leiteiras, uma vez que estão diretamente ligadas a produtividade e saúde dos animais. Uma das grandes dificuldades para o produtor brasileiro é a zona climática a qual o país está inserido, o clima tropical influência a produtividade bovina de leite, devido à ocorrência de altas temperaturas associadas à umidade elevada (Nääs, 1998). Portanto, as instalações utilizadas têm a função de abrigar os animais e criar um microclima com fatores climáticos amenizados, visando assim uma maior produtividade.

Para mensurar o conforto térmico de animais, foram desenvolvidos índices de conforto, o que facilitou a identificação do estresse térmico de forma eficiente. Estes índices de conforto térmico estabelecem um método para que haja a possibilidade de avaliar um determinado ambiente por meio das diversas variáveis climáticas

\section{MATERIAIS E MÉTODOS}

O experimento foi conduzido no setor de bovinos de leite da Fazenda Experimental Lageado, na cidade de Botucatu/SP (22 $51^{\prime} 25,3^{\prime \prime} \mathrm{S}, 48^{\circ} 25^{\prime} 58,2^{\prime \prime}$ O). Inicialmente foi realizado um levantamento das condições ambientais internas do alojamento sem climatização. O rebanho continha 25 vacas holandesas, em lactação. Foram coletadas variáveis ambientais no galpão de descanso das vacas após ordenha, tais como: Temperatura de bulbo seco (Tbs, ${ }^{\circ} \mathrm{C}$ ), Temperatura de globo negro $\left(\mathrm{Tgn},{ }^{\circ} \mathrm{C}\right)$ e Umidade relativa do ar (UR, \%). Utilizouse 4 dataloggers Hobo $^{\circledR}$ (ONSET, 2018) distribuídos em pontos equidistantes a uma altura de aproximadamente 2 metros existentes. Com o uso de tecnologias voltadas à ambiência, várias pesquisas vêm sendo realizadas (ZHANG, CHEN e BOUTABA, 2010; WANG et al., 2015; JONES, 2015), tornando a computação móvel cada vez mais acessível e presente na vida do produtor rural.

Considerada como uma das tendências da tecnologia da informação, a computação móvel tornou-se um recurso essencial à computação; diversos aplicativos são desenvolvidos atualmente para otimizar meios de comunicação e interação digital, fornecendo resultados rápidos e de qualidade (OLIVEIRA JÚNIOR et al., 2015).

Neste contexto, o objetivo do trabalho foi utilizar a ferramenta mobile Orvalho ${ }^{\circledR}$ para cálculo de índices de conforto visando o bem-estar de vacas leiteiras.

(distância mínima e segura aos bovinos), ajustados para armazenar os dados a cada 2 min, para um período de quadro dias, instalados em quadrantes virtualmente representados dentro do galpão. As medidas de temperatura de globo negro foram obtidas por meio de um termistor conectado aos dataloggers Hobo ${ }^{\circledR} \mathrm{e}$ inserido dentro de uma esfera oca pintada externamente com tinta preta fosca. Após a coleta dos dados ambientais, a fim de avaliar as condições de conforto térmico dos animais, o Índice de Temperatura de Globo e Umidade - ITGU (Eq. 1) foi calculado em função da temperatura de ponto de orvalho (Tpo, ${ }^{\circ} \mathrm{C}$, Eq. 2 ). 


$$
I T G U=T_{g n}+0,36 \times T_{p o}+41,5 \quad \text { Eq. } 1
$$

Em que $\mathrm{T}_{\text {gn }}$ : temperatura de globo negro $\left({ }^{\circ} \mathrm{C}\right)$ e $\mathrm{T}_{\mathrm{po}}$ : temperatura de ponto de orvalho $\left({ }^{\circ} \mathrm{C}\right)$.

$$
T_{p o}=\frac{B_{1} \ln \frac{U R}{100} \div+\frac{A_{1} T_{a}}{B_{1}+T_{a}}}{A_{1} \ln \frac{U R}{100} \div \frac{A_{1} T_{a}}{B_{1}+T_{a}}} \quad \text { Eq. } 2
$$

Sendo $T_{a}$ : temperatura do ar $\left({ }^{\circ} \mathrm{C}\right)$, UR: umidade relativa $(\%), \mathrm{A}_{1}=17,625$ e $\mathrm{B}_{1}=$ 243,04, segundo Alduchov e Eskridge (1996).
$\mathrm{O}$ índice de Temperatura e Umidade - ITU para bovinos, segundo Vitali et al., 2009 (Eq. 3), também foi calculado após as medições.

$$
I T U_{\text {bovinos }}=(1,8 \times T a+32)-(0,55-0,55 \times U R)[(1,8 \times T a+32)-58] \quad \text { Eq. } 3
$$

Em que $T_{a}$ é a temperatura do ar $\left({ }^{\circ} \mathrm{C}\right)$ e UR a umidade relativa $(\%)$.

A partir dos dados climatológicos medidos no alojamento dos bovinos de leite, os índices foram calculados por meio do aplicativo Orvalho ${ }^{\circledR}$ para smartphones Android 5.0 ou superior. A Figura 1 exibe a forma de cálculo do índice ITGU.
A.

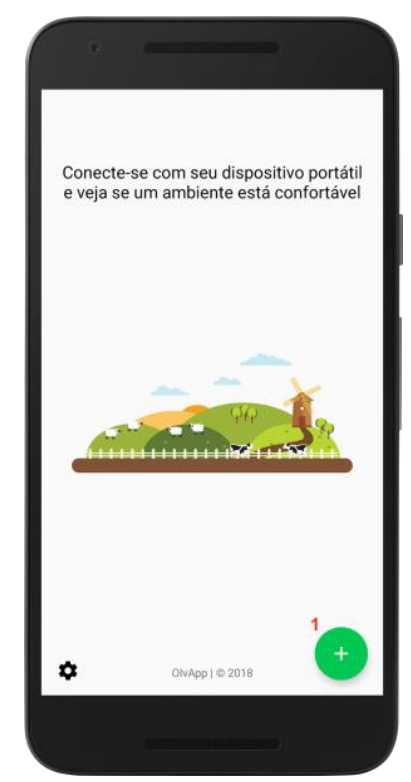

B.

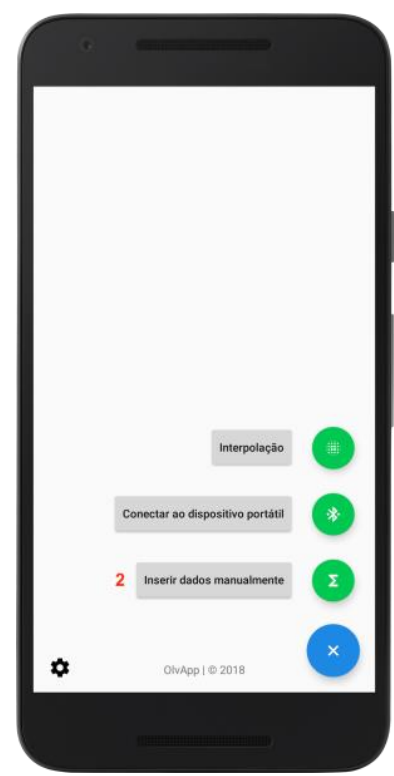

C.

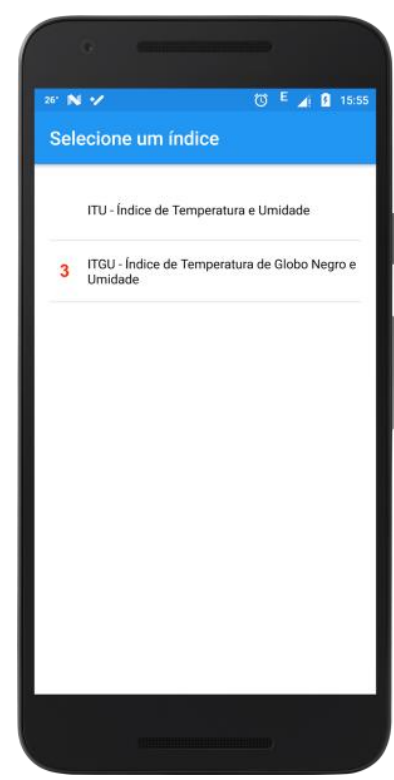

D.

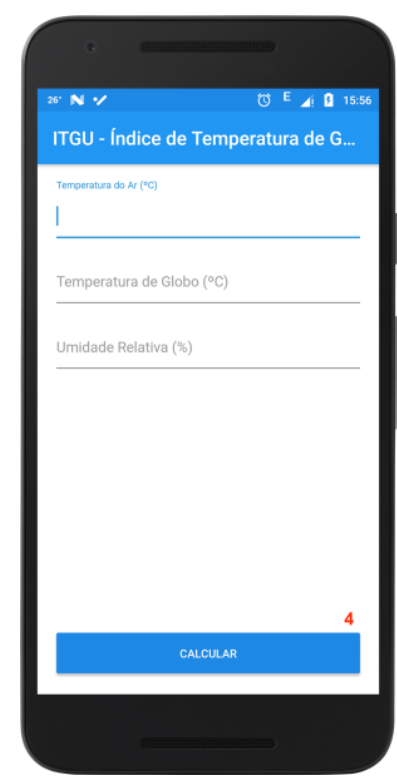

Figura 1. Cálculo do índice ITGU por meio do aplicativo Orvalho ${ }^{\circledR}: 1$. Tela inicial (A.); 2. Escolha de opção de cálculo manual (B.); 3. Escolha do índice ITGU (C.); 4. Inserção de dados e cálculo de índice (D.) 
As delimitações de conforto térmico foram implementadas para o ITGU de acordo com os limites definidos por Baêta e Souza (2010), conforme Tabela 1. Sendo os limites de ITU segundo Armstrong, 1994 (Tabela 2).

Tabela 1. Limites de conforto térmico para bovinos - ITGU

\section{Condição}

Conforto térmico

Estresse térmico (leve)

Estresse térmico (perigo)

\section{ITGU}

ITGU $<74$

$74 \leq$ ITGU $<79$

$79 \leq \mathrm{ITGU}<84$

Tabela 2. Limites de conforto térmico para bovinos - ITU

\begin{tabular}{cc}
\hline Condição & ITU \\
\hline Conforto térmico & ITU $<72$ \\
Estresse pelo calor (brando) & $72 \leq$ ITU $<79$ \\
Estresse pelo calor (moderado) & $79 \leq$ ITU $<89$ \\
Estresse pelo calor (severo) & $89 \leq$ ITU $<98$ \\
\hline
\end{tabular}

\section{RESULTADOS E DISCUSSÃO}

Na Tabela 3 são apresentados os valores mínimos (7h00) e máximos (15h00) da temperatura de bulbo seco e umidade relativa medidos no alojamento sem climatização de bovinos de leite.

Tabela 3. Temperatura do ar, umidade relativa e temperatura de globo negro em alojamento de bovinos de leite da Fazenda Experimental Lageado, medidos durante os dias 26-29² de abril de 2016

\begin{tabular}{ccccccc}
\hline Dias & $\begin{array}{c}\mathrm{T}_{\mathrm{a}}{ }^{\circ} \mathrm{C} \\
(\min )\end{array}$ & $\begin{array}{c}\mathrm{T}_{\mathrm{a}}{ }^{\circ} \mathrm{C} \\
(\max )\end{array}$ & $\begin{array}{c}\mathrm{UR} \% \\
(\min )\end{array}$ & $\begin{array}{c}\mathrm{UR} \% \\
(\max )\end{array}$ & $\begin{array}{c}\mathrm{T}_{\mathrm{gn}}{ }^{\circ} \mathrm{C} \\
(\min )\end{array}$ & $\begin{array}{c}\mathrm{T}_{\mathrm{gn}}{ }^{\circ} \mathrm{C} \\
(\max )\end{array}$ \\
\hline 26/abr & 18,41 & 20,34 & 71,65 & 80,0 & 18,43 & 20,42 \\
$27 / \mathrm{abr}$ & 14,44 & 19,11 & 66,5 & 77,2 & 14,50 & 19,15 \\
28/abr & 11,54 & 23,53 & 52,31 & 78,97 & 11,61 & 23,62 \\
29/abr & 13,54 & 23,47 & 60,03 & 78,29 & 13,59 & 23,52 \\
& & & & & & \\
\hline
\end{tabular}

\footnotetext{
${ }^{2}$ Tempo disponível para coleta de dados na Fazenda Experimental.
} 
Na Figura 2 são apresentadas as medidas mínimas e máximas de Ta, UR e Tgn

A.

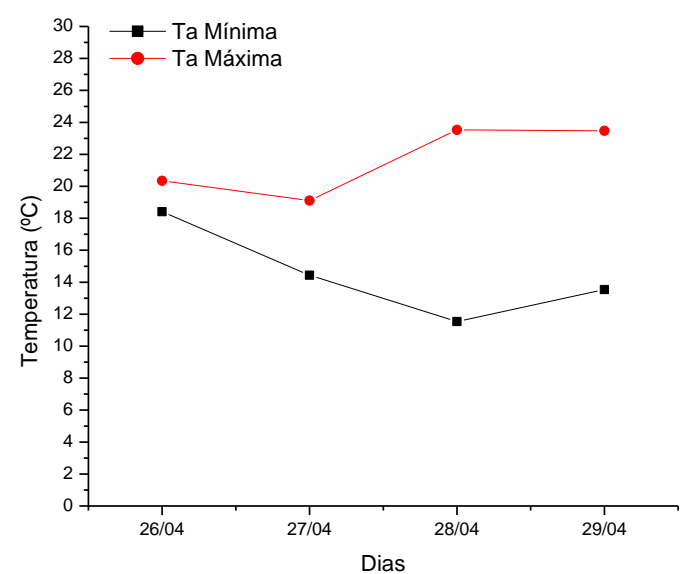

C.

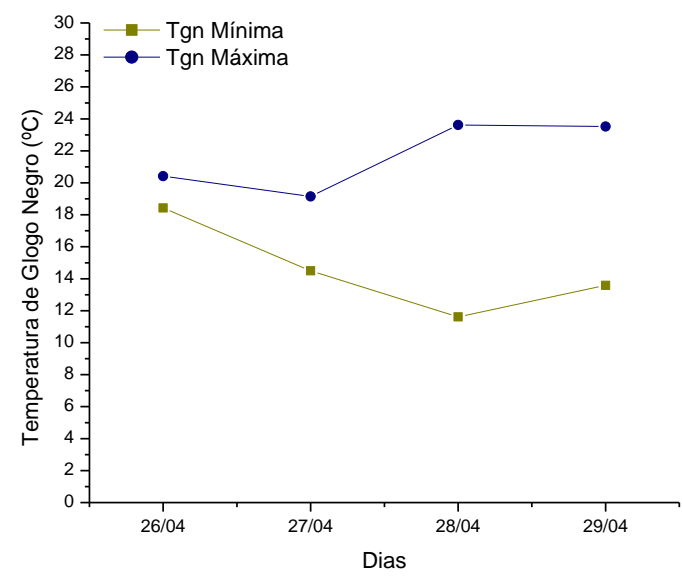

durante o período de coleta de dados, de 26 a 29 de abril.
B.

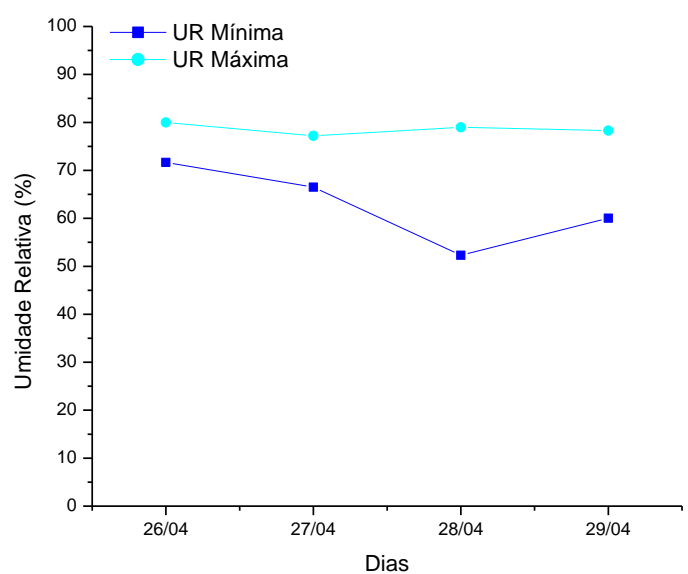

Figura 2. Medidas de temperatura do ar (A.), umidade relativa (B.) e temperatura de globo negro (C.) do alojamento de bovinos de leite, Fazenda Experimental Lageado.

Os índices ITGU e ITU calculados mediante o aplicativo Orvalho $^{\circledR}$, são apresentados na Tabela 4. A Figura 3 ilustra um exemplo de cálculo dos índices para o dia 28 de abril, em que se obteve os maiores valores de ITGU e ITU.

Tabela 4. Valores de ITGU e ITU para bovinos de leite a partir de medidas de máximo e mínimo de Ta, UR e Tgn

\begin{tabular}{ccccc}
\hline Dias & $\begin{array}{c}\text { ITGU } \\
(\min )\end{array}$ & $\begin{array}{c}\text { ITGU } \\
(\text { máx })\end{array}$ & $\begin{array}{c}\text { ITU } \\
(\min )\end{array}$ & $\begin{array}{c}\text { ITU } \\
(\text { máx })\end{array}$ \\
\hline $26 / a b r$ & 64,68 & 67,96 & 64,03 & 67,44 \\
$27 / a b r$ & 58,98 & 66,06 & 57,99 & 65,34 \\
$28 / a b r$ & 53,87 & 72,20 & 54,14 & 72,46 \\
$29 / a b r$ & 57,22 & 72,03 & 56,73 & 72,31 \\
\hline
\end{tabular}



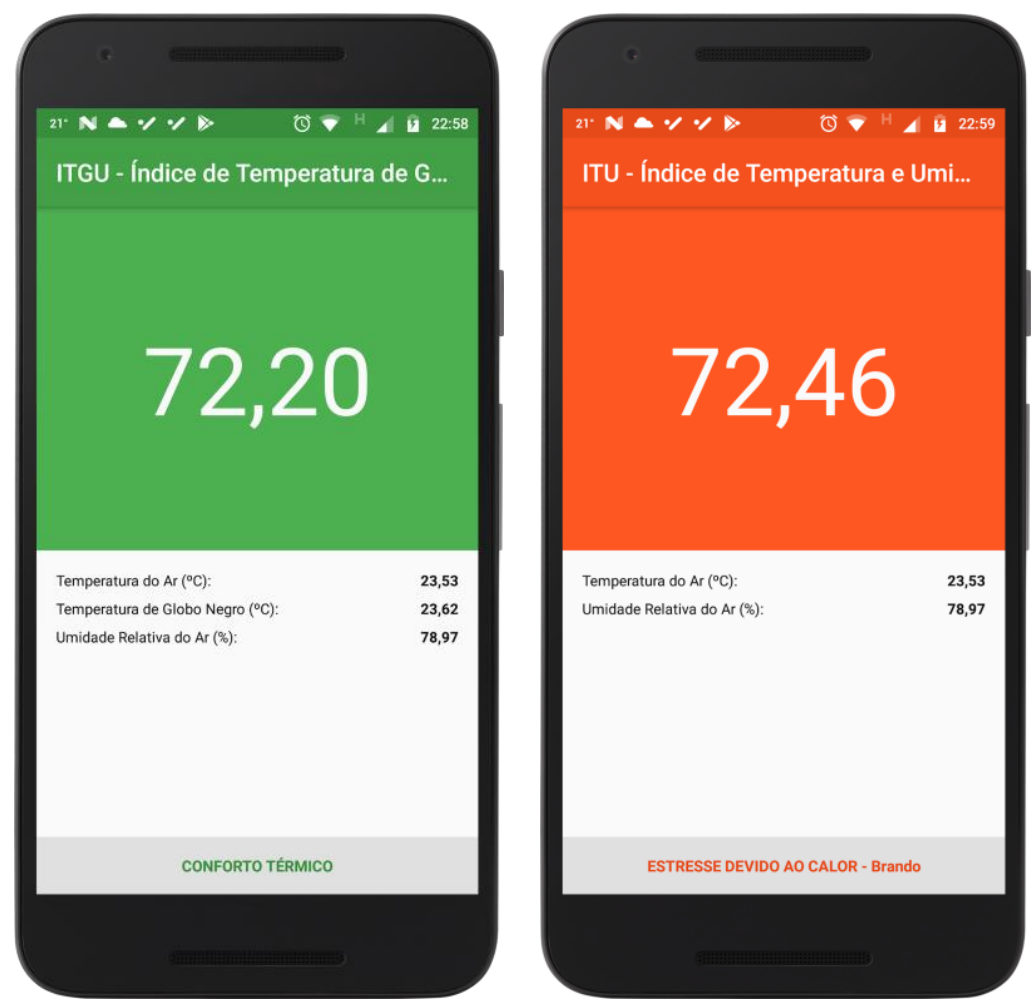

Figura 3. Telas de resultado de cálculo dos índices ITGU e ITU - aplicativo Orvalho ${ }^{\circledR}$

Sabe-se que a temperatura e a umidade relativa apresentam uma relação inversa na atmosfera, todavia para os valores de ITGU e ITU máximos e mínimos foram adotadas condições que podem ser criadas no microclima de alojamentos devido a uma climatização inadequada, como por exemplo, temperatura e umidade relativa elevada.

Os valores apresentados pelo índice ITGU, em que o maior valor obtido foi de 72,03 (ITGU máximo), estão dentro dos limites de conforto térmico para bovinos de leite, segundo Baêta e Souza (2010). Para os dados obtidos pelo ITU houve duas condições de estresse devido ao calor do tipo brando - segundo Armstrong (1994), sendo uma no dia 28 de abril (ITU = $72,46)$ e outra no dia 29 de abril (ITU = 72,31). Vale ressaltar que as condições estabelecidas para esse estado de estresse térmico foram de temperatura e umidade relativa máxima. Tem-se que para o período de lactação os limites ideais de

\section{CONCLUSÕES}

Concluiu-se que os valores apresentados dos índices ITGU e ITU, temperatura devem ser de 4 a $24{ }^{\circ} \mathrm{C}$ (MARTELLO, 2004). Para Broucek (2009), a temperatura máxima considerada crítica para vacas leiteiras é de 24 a $27^{\circ} \mathrm{C}$. Dadas as condições, observa-se que as temperaturas máximas do período estudado estão dentro dos limites considerados ideias para lactação. Ao comparar os resultados de ITU com trabalhos como o de Oliveira et al. (2018), é possível compreender que para o outono as condições climáticas são mais favoráveis para o período de lactação, considerando os valores apresentados para regiões próximas a Botucatu/SP.

O aplicativo Orvalho ${ }^{\circledR}$ auxiliou de forma prática $\mathrm{o}$ cálculo dos índices estudados e demonstrou ser um recurso móvel útil para pequenas avaliações de condições térmicas de bovinos de leite, visto que produtores e pesquisadores que possuem smartphones podem ter acesso ao app que lhes permite verificar as condições térmicas de seus alojamentos in loco.

durante o período de estudo, estavam dentro dos limites de conforto térmico para 
bovinos de leite. $\mathrm{O}$ uso da ferramenta mobile foi prático e eficiente na obtenção

\section{REFERÊNCIAS}

Alduchov, O. A.; Eskridge, R. E. Improved Magnus form approximation of saturation vapor pressure. J. Appl. Meteor., 35, 601609, 1996.

Armstrong, D.V. Heat stress interaction with shade and cooling. Journal of Dairy Science, New York, v.77, p.2044-2050, 1994.

BAÊTA, F. C.; SOUZA, C. F. Ambiência em edificações rurais - conforto animal. Universidade federal de Viçosa, 2010.

BROUCEK, J.; KISAC, P.; UHRINCAT, M. Effect of hot temperatures on the hematological parameters, health and performance of calves. International Journal of Biometeorology, v.15, p.201208, 2009.

JONES, S. Cloud computing procurement and implementation: Lessons learnt from a United Kingdom case study. International Journal of Information Management, Oxford, v. 35, n. 6, p.712-716, dez. 2015. Elsevier BV.

MARTELLO, L. S.; SAVASTANO JUNIOR, H.; LUZ, S.; et al. Respostas fisiológicas e produtivas de vacas Holandesas em lactação submetidas a diferentes ambientes. Revista Brasileira de Zootecnia. v.33, n.1, p.181-191, 2004.

NÃ̃̃S, I. A. Biometeorologia e construções rurais em ambiente tropical. In: CONGRESSO BRASILEIRO DE BIOMETEOROLOGIA, 2. Anais, Goiânia, p. 63-73, 1998. do cálculo dos índices de conforto térmico ITGU e ITU.

OLIVEIRA JÚNIOR, A.; SOUZA, S. R. L.; BARROS, Z. X.; SARTORI, M. M.; FRANCO, L. V. Índice de desconforto e Índice de temperatura efetiva: Uma Implementação para Smartphones e Tablets. Revista Energia na agricultura, v. 30, n.2, p. 165- 163, 2015.

OLIVEIRA, Z. B.; Silva, C. M.; Souza, I. J.; Link, T. T.; Bottega, E. L. CENÁRIOS DE MUDANÇAS CLIMÁTICAS E SEUS IMPACTOS NA PRODUÇÃO LEITEIRA NO SUL DO BRASIL. Brazilian Journal Of Biosystems Engineering, Tupã, v. 12, n. 2, p.110-121, jun. 2018.

ONSET. Hobo U12-012. Disponível em: http://www.onsetcomp.com/products.

Acesso em: 18 maio 2018.

Q. Zhang, L. Cheng, R. Boutaba, Cloud computing: state-of-the-art and research challenges, Journal of Internet Services and Applications, v1 (2010) 7-18.

Vitali, A.; Segnalini, M.; Bertocchini, L.; Bernabuccini, U.; Nardone, A.; Lacetera, N. Seasonal pattern of mortality and relationships between mortality and temperature-humidity index in dairy cows. Journal Dairy Science. v.92, p. 3781-3790, 2009.

WANG, B.; Q, Z.; M, R.; Guan, H.; Vasilakos, A. V. A survey on data center networking for cloud computing. Computer Networks, Amsterdam, v. 91, p.528-547, nov. 2015. Elsevier BV. 